\title{
The preferred level of face categorization depends on discriminability
}

\author{
Christopher D'LaUro \\ University of Colorado, Boulder, Colorado \\ JAMES W. TANAKA \\ University of Victoria, Victoria, British Columbia \\ AND \\ Tim CurRaN \\ University of Colorado, Boulder, Colorado
}

\begin{abstract}
People usually categorize objects more quickly at the basic level (e.g., "dog") than at the subordinate (e.g., "collie") or superordinate (e.g., "animal") levels. Notable exceptions to this rule include objects of expertise, faces, or atypical objects (e.g., "penguin," "poodle"), all of which show faster than normal subordinate-level categorization. We hypothesize that the subordinate-level reaction time advantage for faces is influenced by their discriminability relative to other faces in the stimulus set. First, we replicated the subordinate-level advantage for faces (Experiment 1) and then showed that a basic-level advantage for faces can be elicited by increasing the perceptual similarity of the face stimuli, making discrimination more difficult (Experiment 2). Finally, we repeated both effects within subjects, showing that individual faces were slower to be categorized in the context of similar faces and more quickly categorized among diverse faces (Experiment 3 ).
\end{abstract}

For average observers, the basic level of categorization is the nexus of their knowledge about visual objectsboth the level of abstraction where they primarily organize information about a class of objects and the most inclusive category level at which members can be visually identified by their averaged shape (Rosch, Mervis, Gray, Johnson, \& Boyes-Braem, 1976). The basic level can be thought of as the optimal level for differentiating objects. Because of this structural distinctiveness, it is easier to differentiate stimuli at the basic level (e.g., "dog" vs. "cat") than at the subordinate level (e.g., "Dalmatian" vs. "collie"); and the basic level provides more information about an exemplar than does its superordinate-level description (e.g., "animal"). Thus, the basic level offers the optimal trade-off for maximizing an object's "discriminability" and "specificity" (Murphy \& Brownell, 1985).

The basic level has been identified as the entry point in visual object recognition - that is, the point at which the perceptual stimulus makes initial contact with a stored visual representation (Jolicœur, Gluck, \& Kosslyn, 1984). For instance, Rosch et al. (1976) showed that people are quickest to classify typical objects at the basic level (e.g., "dog") and slowest to identify them at the subordinate ("Dalmatian") and superordinate ("living thing") levels. Subsequent studies have confirmed the basic-level advantage in recognition (Murphy \& Brownell, 1985;
Tanaka, 2001; Tanaka \& Taylor, 1991) and shown that subordinate-level categorizations require additional perceptual processing (Grill-Spector \& Kanwisher, 2005), whereas superordinate-level categorizations require semantic processing (Jolicœur et al., 1984).

However, the basic-level pattern is not without exceptions. Atypical category exemplars (e.g., "ostriches," "poodles") that deviate in their physical appearance from the category prototype are categorized more quickly at the subordinate level than at the basic level (Jolicour et al., 1984). Other work has similarly shown that atypical faces have recognition advantages over typical faces (Bartlett, Hurry, \& Thorley, 1984; Light, Kayra-Stuart, \& Hollander, 1979; Tanaka \& Corneille, 2007; Vokey \& Read, 1992). Expertise can also produce a subordinate-level shift in recognition where experts are as quick to identify objects in their domain of expertise at the subordinate level as at the basic level (Tanaka \& Taylor, 1991). Similarly, as "face experts," people are as fast to identify familiar faces at the subordinate level of the individual ("Tom Cruise") as they are at the basic level ("human") (Tanaka, 2001).

How can a single theory account for these varied levels of entry point? According to Murphy and Brownell's (1985) differentiation hypothesis, differentiation of an exemplar (a combined function of its distinctiveness and specificity) is the key to object identification. Easily dis-

C. D'Lauro, christopher.dlauro@colorado.edu 
tinguished categories (e.g., "dog" vs. "cat") typically lack specificity and may fail to provide some key predictive information (e.g., "pit bull" vs. "poodle"); meanwhile, more specific categories carry better predictive information but are less discriminable. To optimize both discriminability and specificity, people attempt to retrieve as much information as possible. But discriminability of stimuli can be increased in two obvious ways: The stimuli themselves can be more different from each other (e.g., atypical exemplars), or the observer can be more skilled at differentiating exemplars (e.g., the bird expert). Thus, discriminability is jointly determined both by the perceiver's ability to discriminate stimuli and by the stimulus's structural distinctiveness relative to other within-category exemplars.

This view is supported also by recent computational work performed by Mack, Wong, Gauthier, Tanaka, and Palmeri (2007), who implemented an exemplar-based random walk (EBRW) model to compare categorizations of novice (dog, bird) and expert (faces) stimuli. According to EBRW, categorization performance depends on the relative similarity of a test object to object representations stored in memory. Simulations showed that in recognition, objects can be perceived as more distinct for at least two reasons: Either they are physically more different from other category members (i.e., atypicality effects), or they are more psychologically discriminable because of the observer's sensitivity to remembering perceptual details (i.e., expertise effects).

When pitted against one another, the effects of structural similarity can sometimes hold sway over the influences of perceptual expertise. For example, Robbins and McKone (2003) showed that, despite extensive training, subjects were still faster and more accurate at categorizing pictures of identical twins at the more general level of family and gender than at the subordinate level of identity. Although it is not surprising that the subordinate-level shift would be eliminated in the extreme case of recognizing identical twins, it is less certain whether structural similarity affects the processes of normal face recognition. To address this question, we examined the recognition of familiar celebrity faces in the context of low within-category similarity (Experiment 1), high within-category similarity (Experiment 2), and a mixture of low and high within-category similarity (Experiment 3). If face expertise is not affected by the structural similarity of competing within-category faces, we expected a subordinate-level advantage regardless of the test context. Alternatively, if face expertise is sensitive to within-category similarity, the subordinatelevel shift is more likely to be found under conditions of low within-category similarity as opposed to high withincategory similarity. In Experiment 1, we directly replicated Tanaka's (2001) study using the same heterogeneous set of famous faces.

\section{EXPERIMENT 1}

\section{Method}

Subjects. Twenty-two University of Colorado undergraduates volunteered to participate in this experiment for class credit. Sub- jects had normal or corrected-to-normal vision. In this and all subsequent experiments, subjects were asked questions that enabled the exclusion of dog experts, though none had to be excluded.

Materials. The stimuli were color photographs of eight celebrities, eight dogs, and eight inanimate objects, all with white backgrounds. The stimuli were roughly equivalent in area and were equilibrated for luminosity within Adobe Photoshop. The celebrity and dog pictures were photographs (from Tanaka, 2001) of Cindy Crawford, George Bush Sr., Jerry Seinfeld, Bill Clinton, Hillary Clinton, Marilyn Monroe, Jay Leno, and Princess Diana; beagle, chow, collie, Doberman pinscher, German shepherd, poodle, retriever, and terrier (see Figure 1). Subjects were familiarized with all dogs and faces before testing by being shown each one along with the corresponding names. The inanimate comparison objects for the superordinatelevel comparisons were a saw, a guitar, a hammer, a lamp, a drum, a car, a screwdriver, and a table. All stimulus presentations and data recordings were administered through E-Prime (Psychology Software Tools, Inc., Pittsburgh, PA).

Design and Procedure. In the category verification task, subjects were first presented with a category name, such as "dog," "human," or "Jerry Seinfeld," and were presented a picture shortly afterward; their task was to indicate as quickly and as accurately as possible whether the category and the picture matched. Each experimental trial consisted of (1) an initial central fixation stimulus (+) for $500 \mathrm{msec}$; (2) a category label for $255 \mathrm{msec}$; (3) another central fixation plus sign for between 500 and 1,000 msec, varied randomly; (4) the test picture appearing for $255 \mathrm{msec}$; and (5) a poststimulus fixation plus sign until the subject responded by pressing a button for true or false. After the response was recorded, the central fixation point changed to an equal sign $(=)$ to alert the subject that the response had been received. The next trial would start after a 1 -sec pause. Left/right key assignment was counterbalanced across subjects.

Each subject completed two blocks of 128 trials in which each stimulus was presented six times, once at each category level (subordinate, basic, superordinate) and in each response condition (true or false). For example, true trials for Hillary Clinton included the preceding category labels of "living thing," "human," and "Hillary Clinton" at the superordinate, basic, and subordinate levels, respectively. For false trials, the same image of Hillary Clinton was preceded by the labels "nonliving thing," "dog," and "Marilyn Monroe," for superordinate, basic, and subordinate levels, respectively. Subordinate false labels were chosen randomly from among other category exemplars.

\section{Results and Discussion}

The primary dependent measure was reaction time (RT) on correct true trials, because these trials resulted in positive identification of the stimuli at the designated category level, whereas correct false trials could be the result of stimuli being excluded from category identification. (For completeness, RT and accuracy results from false trials appear in the Appendix.) Mean RT and accuracy are displayed by stimulus type and category level in Figure 2.

RTs on correct true trials were analyzed using a 3 (category level: subordinate, basic, superordinate) $\times 2$ (stimulus type: face, dog) ANOVA. Category level and stimulus type interacted significantly $\left[F(2,42)=3.84, M S_{\mathrm{e}}=4,636.6\right.$, $p<.05]$, showing an RT cost at the subordinate level for dog trials, but not for face trials. A subsequent means test confirmed that dog trials showed a significant RT cost from the basic to subordinate level $(p<.01)$, but face trials did not $(p=.53)$. Accuracy was high for both dogs $(96.9 \%)$ and faces (97.2\%). A 3 (category level: subordinate, basic, superordinate) $\times 2$ (stimulus type: face, $\operatorname{dog}$ ) ANOVA of true trials did not reveal significant effects. 
Experiment 1
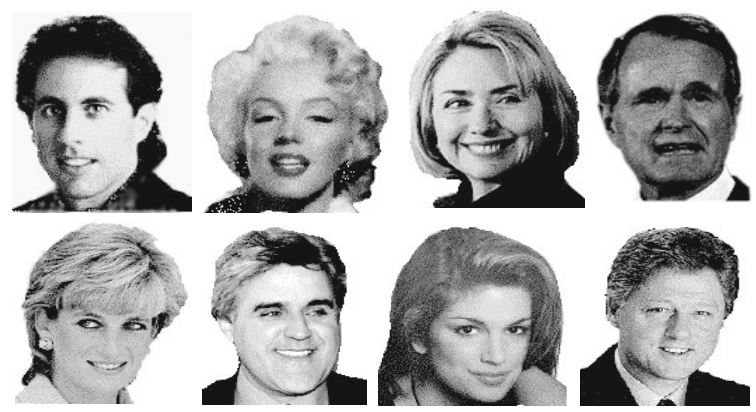

Experiments 1, 2,3
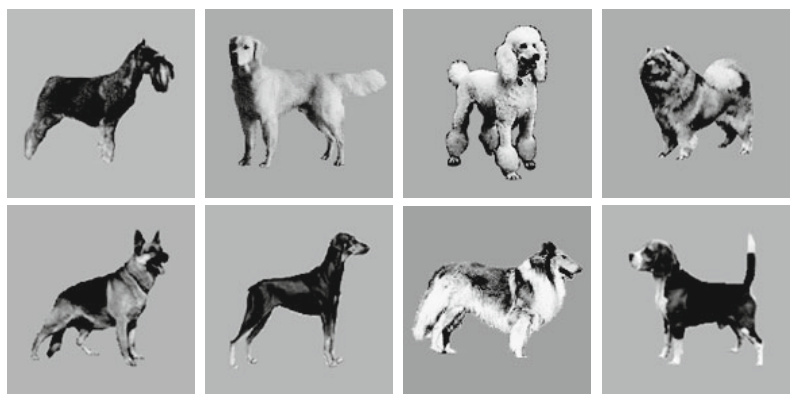

Experiments 2, 3 (homogeneous block)
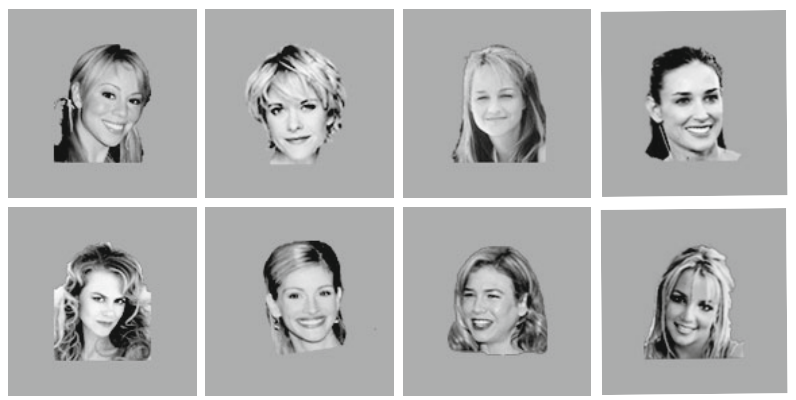

Experiment 3 (diverse block)
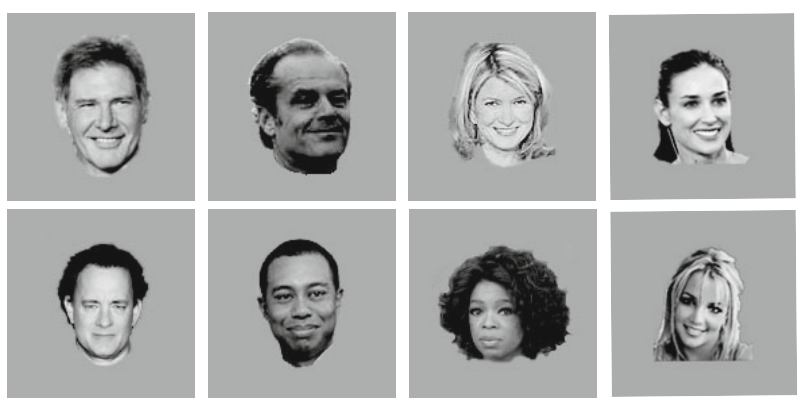

Figure 1. Grayscale versions of the test picture stimuli used in Experiments 1-3; color versions were used in testing. The dogs were used in Experiments 1-3 (except for the poodle, which was used in Experiments 2 and 3 after replacing a drawing used in Experiment 1). In Experiment 1, the dog pictures were set against a white background. Demi Moore and Britney Spears, pictured in the last column of the bottom face sets, were included in both the homogeneous and diverse blocks in Experiment 3.

\section{EXPERIMENT 2}

Experiment 1 replicated Tanaka (2001) by showing a basic-level RT advantage for dogs but a subordinate-level shift for faces. In Experiment 2, we tested the differentiation hypothesis by changing the face stimuli to include only female entertainers, making them less perceptually discriminable. In previous experiments with homogeneous face sets, subjects showed poorer performance on subordinate-level face tasks (Grill-Spector \& Kanwisher, 2005; Robbins \& McKone, 2003). These results make sense in terms of the differentiation hypothesis, which would predict that extremely similar faces should not show a subordinate-level shift.

\section{Method}

Subjects. Thirty-three University of Colorado undergraduates volunteered to participate in Experiment 2 for class credit. Subjects had normal or corrected-to-normal vision.

Materials. The stimuli consisted of those in Experiment 1, except for the face pictures, which were changed to be more similar to each other, and the poodle drawing, which was exchanged for a more realistic picture. The face stimuli included pictures of Britney Spears, Demi Moore, Julia Roberts, Mariah Carey, Renee Zellweger, Meg Ryan, Helen Hunt, and Nicole Kidman. The new faces were selected from a larger set that was pretested for subordinate-level naming; faces in this experiment averaged $77 \%$ identification (range $=$ $57 \%-93 \%, n=28$ ). All were placed against a constant gray $140 \times$ 140 pixel background (see Figure 1).

Design and Procedure. The experimental trials and block setups were identical to these in Experiment 1; however, subjects of Experiment 2 completed six blocks, for 864 total trials. EEG was recorded, but the data are not presented here.

\section{Results and Discussion}

Mean RT and accuracy are displayed by stimulus type and category level in Figure 2. True, correct trial RTs were analyzed using a 3 (category level: superordinate, basic, subordinate) $\times 2$ (stimulus type: face, $\operatorname{dog}$ ) ANOVA. As predicted, there was a significant main effect only of level $\left[F(2,64)=17.2, M S_{\mathrm{e}}=3,346.06, p<.0001\right]$. The interaction of level and stimulus type was nonsignificant $(p=.096)$ and trended in the direction opposite that of Experiment 1, with subordinate dog trials having shorter RTs than subordinate face RTs $(p=.11)$.

For accuracy, category level and stimulus type interacted significantly $\left[F(2,64)=11.9, M S_{\mathrm{e}}=0.001, p<.0001\right]$, with subordinate true face trials showing poorer accuracy than subordinate true dog trials despite similar accuracy for true face and dog trials at the basic and superordinate levels. This lower accuracy in the true subordinate face trials may also speak to the difficulty of categorizing the more similar faces of Experiment 2.

\section{EXPERIMENT 3}

Experiment 1 reproduced the RT results obtained by Tanaka (2001), and Experiment 2 used less discriminable faces to produce a basic-level face advantage, as would be predicted by the differentiation hypothesis (Murphy \& Brownell, 1985). For Experiment 3, subjects completed 

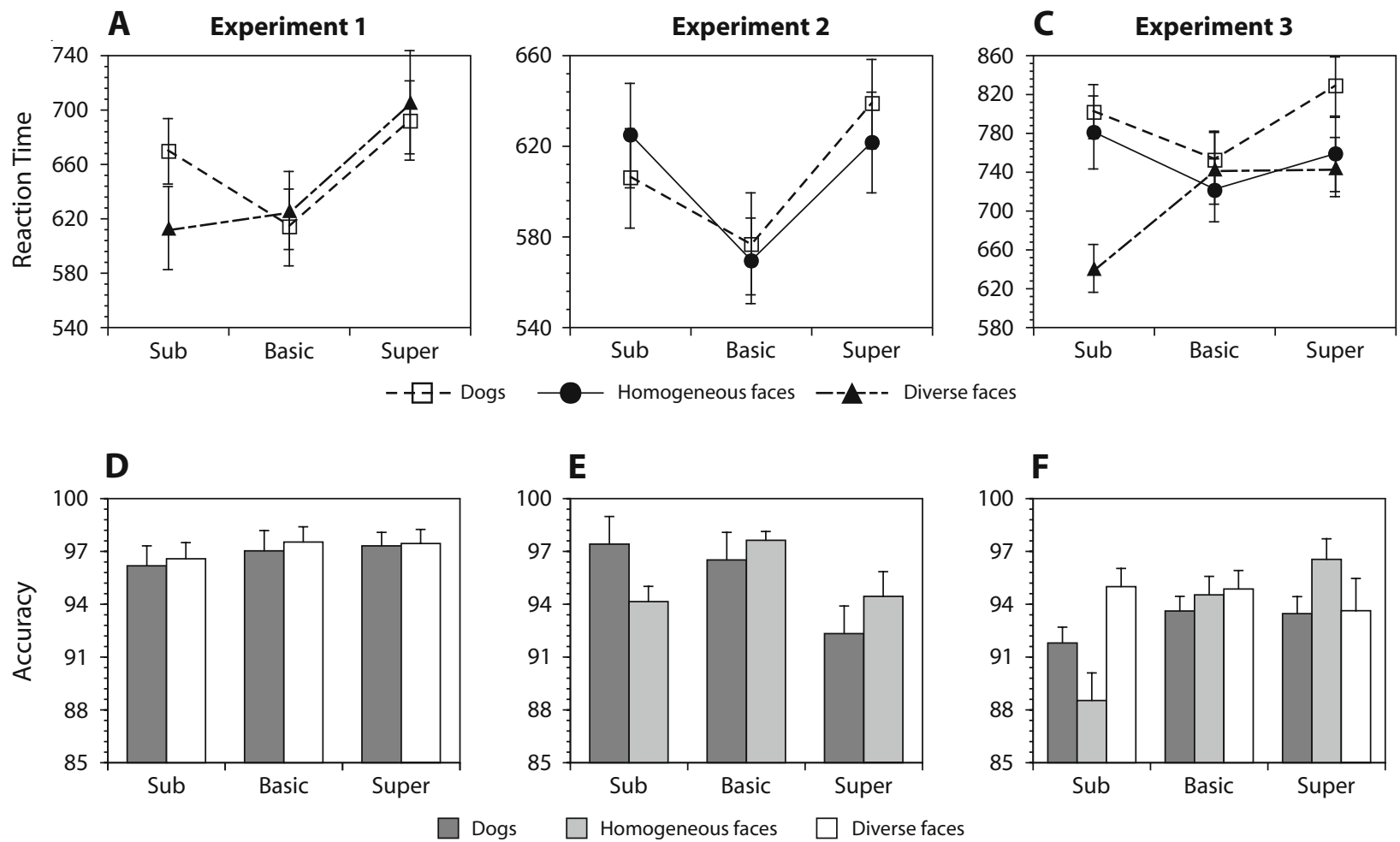

Figure 2. Mean reaction times (RTs, in milliseconds) and accuracy (percentage correct) for true trials in Experiments 1-3 by stimulus type and category level (subordinate [Sub], basic, superordinate [Super]). Error bars are standard errors of the means. (A) RT for dogs and original Tanaka (2001) faces (Experiment 1). (B) RT for dogs and homogeneous faces (Experiment 2). (C) RT for homogeneous faces, diverse faces, and dogs (Experiment 3). (D) Accuracy for dogs and original Tanaka faces (Experiment 1). (E) Accuracy for dogs and homogeneous faces (Experiment 2). (F) Accuracy for homogeneous faces, diverse faces, and dogs (Experiment 3).

two blocks of trials, one with the homogeneous set of faces from Experiment 2 and another with diverse faces like those in Experiment 1. If the context of similar faces in Experiment 2 was causing the basic-level advantage, we would expect to show the basic-level advantage for faces in homogeneous blocks and the subordinate-level shift for faces in the diverse block. Furthermore, we exclude explanations that rely on stimulus characteristics other than similarity (e.g., differing fame of celebrities) by including two faces in both the homogeneous and the heterogeneous blocks. If the context of the other faces causes differences between the blocks, we would expect subordinate-level RT to these faces to be faster when they appear in the diverse than in the homogeneous blocks.

\section{Method}

Subjects. Participants in this experiment included 52 University of Colorado undergraduate volunteers; 8 other subjects were excluded for low accuracy or for being nonnative speakers who were not familiar with the celebrities. Subjects were counterbalanced for handedness and block order. Subjects had normal or corrected-tonormal vision.

Materials. Because many of the celebrity faces used in the Tanaka (2001) study were no longer current, a new set of faces that included a similar range of ages and genders was collected. These faces included Tiger Woods, Harrison Ford, Tom Hanks, Martha Stewart, Oprah Winfrey, and Jack Nicholson. These faces, along with Demi Moore and Britney Spears, comprised the "diverse" face block (Figure 1). The "homogeneous" block consisted of the face ex- emplars from Experiment 2. Thus, Demi Moore and Britney Spears were included in both blocks, but performance on these stimuli was expected to differ between blocks.

Design and Procedure. In this experiment, subjects completed the same task in both diverse and homogeneous face blocks (order counterbalanced across subjects) with dog and object stimuli kept constant, essentially performing a within-subjects version of Experiments 1 and 2. Subjects performed 92 trials in each block, due to a reduced number of superordinate trials.

\section{Results and Discussion}

Mean RT and accuracy are displayed by stimulus type and category level in Figure 2. Mean RTs of correct true trials were analyzed through a 3 (category level: subordinate, basic, superordinate) $\times 2$ (stimulus type: dog, face) $\times 2$ (face context: diverse, homogeneous) ANOVA. The predicted interaction of level, stimulus type, and face context block was significant $\left[F(2,102)=3.853, M S_{\mathrm{e}}=\right.$ $15,916, p<.05$ ], showing slower RT judgments for faces at the subordinate level in the homogeneous block (see Figure 2). This interaction subsumed significant main effects of stimulus type, block, and level. A subsequent planned $t$ test of face RTs further demonstrated that basiclevel judgments were faster than subordinate ones in the homogeneous block $(p<.05)$, whereas the reverse pattern was true in the diverse face block $(p<.05)$.

Accuracy of true trials was analyzed through a 3 (category level: subordinate, basic, superordinate) $\times 2$ (stim- 
ulus type: dog, face) $\times 2$ (face context: diverse, homogeneous) ANOVA (see Figure 2). There was a significant interaction of stimulus, level, and block $[F(2,102)=4.67$, $\left.M S_{\mathrm{e}}=0.012, p=.01\right]$, such that accuracy was considerably lower for subordinate-level faces in the homogeneous than in the diverse blocks, another indicator of this condition's greater difficulty. Overall, subjects showed high accuracy for both dogs (93.0\%) and faces (93.9\%).

As it stands, one could argue that subjects are simply quicker to verify the better known diverse faces than the less well-known homogeneous ones. To negate this argument, we included two faces in both blocks: Britney Spears and Demi Moore. If faces of more famous people were simply verified more quickly, we would expect these faces to show the same RTs in both blocks, but this was not the case. These RTs are displayed in Figure 3 by block and level. Instead, we found a significant block $\times$ level interaction $\left[F(1,51)=5.2, M S_{\mathrm{e}}=35,992\right.$, $p=.03$ ], showing an RT cost only in the subordinate trials of the homogeneous block. Planned $t$ tests confirmed subordinate-level RT costs in the homogeneous block ${ }^{1}$ $(p=.03)$ but showed no difference between basic-level and subordinate-level RTs in the diverse block. Thus, the context of the other faces, not some property particular to the chosen faces, must have caused the differences between blocks.

\section{GENERAL DISCUSSION}

Tanaka (2001) showed that the basic-level advantage in the categorization of common objects, such as dogs, differs from the categorization of faces, in which performance can be equivalent at the basic and subordinate levels (the "subordinate-level shift"). These results suggested that preferred categorization levels are an inherent property of different classes of stimuli. However, other research has demonstrated that expertise can induce a subordinatelevel shift for nonface objects (Tanaka \& Taylor, 1991). Conversely, here we have shown that even faces can show a basic-level advantage if stimulus discriminability is sufficiently difficult.

The present results can be understood in terms of Murphy and Brownell's (1985) differentiation hypothesis, which argued that normally the basic level is the preferred "entry point" because it balances specificity of information and distinctiveness of stimuli. In exceptional cases, where "basic-first" does not hold, subjects show subordinate-level shifts to two types of objects: faces (Tanaka, 2001) and objects of expertise (Tanaka \& Taylor, 1991). In both cases, observers found the stimuli more discriminable than normal objects and were also more likely to spontaneously identify these stimuli at the subordinate level rather than at the basic level.

Although this hypothesis readily explains both expertise-based subordinate-level shifts and atypicality effects, it should be noted that different processes produce these two phenomena. Atypicality effects are driven by structural or intrinsic stimulus distinctiveness, essentially making the kind of manipulation used in this experiment impossible. After all, if similar contrast exemplars were

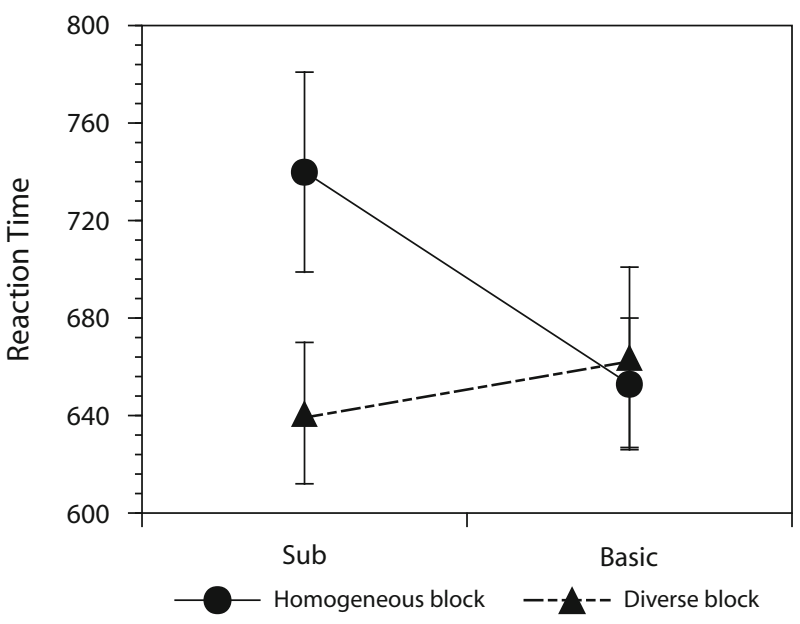

Figure 3. Mean reaction time (in milliseconds) for true trials with Britney Spears and Demi Moore in Experiment 3 by block (diverse or homogeneous faces) and category level (subordinate [Sub] or basic). Error bars are standard errors of the means.

available, the stimuli would not be atypical. For the visual expertise-based shifts, similarity and distinctiveness are relative to the observer. Although category level provides a rough constraint on low-level perceptual qualities, individual differences are still likely in the perceived similarity of exemplars of a category, based both on underlying traits and particularly on prior experience. For example, an extremely adept celebrity watcher might find our "homogeneous" group not particularly difficult to discriminate, although our experiments demonstrate that the average observer has more difficulty. One can imagine similar experience-based differences for discriminating virtually every type of natural stimulus.

Current work in face recognition often describes faces in terms of a perceptual similarity space where more similar faces are clustered together and dissimilar faces are spaced farther apart (Valentine, 1991), which is how we can conceive of the "homogeneous" and "diverse" groups in this experiment. The homogeneous group's faces are bunched together, making them easier to mistake for each other and more effortful to accurately discriminate; meanwhile, people more easily identify the more spatially dispersed, "diverse" face set. Thus, the ability to distinguish a face is not determined by its absolute location in face space, but by the relative proximity of competing face distractors.

This description of our data also accords well with recent computational analyses of categorization and entrylevel phenomena (Mack et al., 2007). Mack et al. used a signal-to-respond procedure to quantify the time course of basic and subordinate categorization of faces, dogs, and birds. Face accuracy data showed quantitative, not qualitative, differences from other stimulus types, a finding similar to the present conclusions. The EBRW model could accurately predict face and object data without making special stimulus allowances. Crucially, this model relied on both a memory-sensitivity parameter and perceptual space differences to model this effect. 
As a final aside, data from Experiment 3 show that faces in the diverse block can be recognized more quickly at the individual level than at the basic level. Although previous studies on faces and other objects of expertise (Tanaka, 2001; Tanaka \& Taylor, 1991) demonstrated equivalent basic- and subordinate-level RTs for faces, there is reason to think that this result is not anomalous. On the basis of the present findings, we might expect that a group of faces that was more diverse than average would show a subordinate-level advantage, given that identity is the most useful information related to a face. The subordinate-level advantage for faces is all the more impressive when one considers that faces are recognized at the most subordinate level of the individual face.

These findings have some relevance for the study of visual expertise, because a subordinate-level shift is often taken as evidence of achieving visual expertise (Gauthier \& Tarr, 1997). The present experiments encourage a view of entry point as an interaction of a stimulus set's perceptual discriminability and observer characteristics. Because these qualities vary independently of each other, the subordinate-level shift may still be a valuable index in training studies where discriminability can be held constant, but less so for natural expertise, where both discriminability and observer sensitivity may vary. Consistent with Murphy and Brownell's (1985) differentiation hypothesis, these experiments argue for a more generalist approach to object and face categorization where the entry point of recognition is jointly determined by interactions between the expertise of the perceiver and the structure of the stimulus environment (Jolicœur et al., 1984; Nosofsky \& Palmeri, 1997; Rosch et al., 1976; Tanaka \& Curran, 2001; Tanaka \& Taylor, 1991).

\section{AUTHOR NOTE}

This research was funded by the Temporal Dynamics of Learning Center (NSF Grant SBE-0542013) and a 21st Century Collaborative Activity Award from the James S. McDonnell Foundation. We thank Brion Woroch and Casey DeBuse for programming and research assistance and members of the Perceptual Expertise Network for helpful input. We particularly thank Thomas Palmeri for his input on perceptual categorization models We also thank Randy O'Reilly, Yuko Munakata, and Lew Harvey for feedback and suggestions. Correspondence concerning this article should be addressed to C. D'Lauro, Department of Psychology, University of Colorado, Meunzinger D244, Boulder, CO 80309-0345 (e-mail: christopher .dlauro@colorado.edu).

\section{REFERENCES}

Bartlett, J. C., Hurry, S., \& Thorley, W. (1984). Typicality and familiarity of faces. Memory \& Cognition, 12, 219-228.

Gauthier, I., \& TARR, M. J. (1997). Becoming a "greeble" expert: Exploring mechanisms for face recognition. Vision Research, 37, 1673-1682.

Grill-Spector, K., \& Kanwisher, N. (2005). Visual recognition: As soon as you know it is there, you know what it is. Psychological Science, 16, 152-160.

Jolicceur, P., Gluck, M. A., \& Kosslyn, S. M. (1984). Pictures and names: Making the connection. Cognitive Psychology, 16, 243-275.

Light, L. L., Kayra-Stuart, F., \& Hollander, S. (1979). Recognition memory for typical and unusual faces. Journal of Experimental Psychology: Human Learning \& Memory, 5, 212-228.

Mack, M. L., Wong, A. C.-N., Gauthier, I., TANaKa, J. W., \& Palmeri, T. J. (2007). Unraveling the time-course of perceptual categorization: Does fastest mean first? In D. S. McNamara \& J. G. Trafton (Eds.), Proceedings of the 29th Annual Conference of the Cognitive Science Society (pp. 1253-1258). Mahwah, NJ: Erlbaum.

Murphy, G. L., \& Brownell, H. H. (1985). Category differentiation in object recognition: Typicality constraints on the basic category advantage. Journal of Experimental Psychology: Learning, Memory, \& Cognition, 11, 70-84.

Nosofsky, R. M., \& PALMeri, T. J. (1997). An exemplar-based random walk model of speeded classification. Psychological Review, 104, 266-300.

Robbins, R., \& McKone, E. (2003). Can holistic processing be learned for inverted faces? Cognition, 88, 79-107.

Rosch, E., Mervis, C. B., Gray, W. D., Johnson, D. M., \& BoyesBraem, P. (1976). Basic objects in natural categories. Cognitive Psychology, 8, 382-439.

TANAKA, J. W. (2001). The entry point of face recognition: Evidence for face expertise. Journal of Experimental Psychology: General, 130, 534-543.

TAnaka, J. W., \& Corneille, O. (2007). Typicality effects in face and object perception: Further evidence for the attractor field model. Perception \& Psychophysics, 69, 619-627.

TANaKa, J. W., \& Curran, T. (2001). A neural basis for expert object recognition. Psychological Science, 12, 43-47.

TANAKA, J. W., \& TAYLOR, M. (1991). Object categories and expertise: Is the basic level in the eye of the beholder? Cognitive Psychology, 23, 457-482.

Valentine, T. (1991). A unified account of the effects of distinctiveness, inversion, and race in face recognition. Quarterly Journal of Experimental Psychology, 43A, 161-204.

VoKey, J. R., \& ReAD, J. D. (1992). Familiarity, memorability, and the effect of typicality on the recognition of faces. Memory \& Cognition, 20, 291-302.

\section{NOTE}

1. This $t$ test remained significant when tested with $\log \mathrm{RT}(p=.03)$. 


\section{APPENDIX}

Table A1

Reaction Time (RT, in Milliseconds) and Accuracy (\% Correct) Data for False Trials in Experiment 1

\begin{tabular}{lccccc}
\hline \multirow{2}{*}{\multicolumn{1}{c}{ Condition }} & \multicolumn{2}{c}{ RT } & & \multicolumn{2}{c}{$\%$ Correct } \\
\cline { 2 - 3 } \cline { 6 - 7 } & $M$ & $S E$ & & $M$ & $S E$ \\
\hline Basic, dog & 719.29 & 38.71 & & 96.7 & .012 \\
Basic, human & 698.37 & 29.57 & & 97.4 & .010 \\
Subordinate, dog & 752.70 & 34.03 & & 96.1 & .010 \\
Subordinate, human & 680.92 & 30.56 & & 96.5 & .011 \\
Superordinate, dog & 956.93 & 47.58 & & 91.0 & .020 \\
Superordinate, human & 921.61 & 53.36 & & 90.4 & .021 \\
\hline
\end{tabular}

Table A2

Reaction Time (RT, in Milliseconds) and Accuracy (\% Correct) Data for False Trials in Experiment 2

\begin{tabular}{lccccc}
\hline \multirow{2}{*}{\multicolumn{1}{c}{ Condition }} & \multicolumn{2}{c}{ RT } & & \multicolumn{2}{c}{ \% Correct } \\
\cline { 2 - 3 } & $M$ & $S E$ & & $M$ & $S E$ \\
\hline Basic, dog & 616.91 & 18.54 & & 96.7 & .006 \\
Basic, human & 611.15 & 16.55 & & 97.5 & .007 \\
Subordinate, dog & 585.56 & 16.21 & & 97.9 & .005 \\
Subordinate, human & 580.51 & 17.36 & & 98.8 & .005 \\
Superordinate, dog & 786.56 & 24.42 & & 92.7 & .008 \\
Superordinate, human & 761.50 & 25.70 & & 94.0 & .010 \\
\hline
\end{tabular}

Table A3

Reaction Time (RT, in Milliseconds) and Accuracy (\% Correct) Data for False Trials in Experiment 3

\begin{tabular}{|c|c|c|c|c|}
\hline \multirow[b]{2}{*}{ Condition } & \multicolumn{2}{|c|}{ RT } & \multicolumn{2}{|c|}{$\%$ Correct } \\
\hline & $M$ & $S E$ & $M$ & $S E$ \\
\hline Homogeneous, basic, dog & 735.34 & 24.70 & 94.2 & .012 \\
\hline Homogeneous, basic, human & 717.16 & 26.62 & 94.5 & .010 \\
\hline Homogeneous, subordinate, dog & 789.88 & 27.90 & 91.8 & .011 \\
\hline Homogeneous, subordinate, human & 777.09 & 29.07 & 88.5 & .016 \\
\hline Homogeneous, superordinate, dog & 815.89 & 31.39 & 91.7 & .017 \\
\hline Homogeneous, superordinate, human & 752.17 & 30.77 & 96.6 & .012 \\
\hline Diverse, basic, dog & 707.47 & 24.80 & 93.0 & .011 \\
\hline Diverse, basic, human & 675.05 & 20.85 & 94.9 & .010 \\
\hline Diverse, subordinate, dog & 767.16 & 25.01 & 91.8 & .013 \\
\hline Diverse, subordinate, human & 623.93 & 19.49 & 95.0 & .010 \\
\hline Diverse, superordinate, dog & 781.14 & 27.37 & 95.3 & .015 \\
\hline Diverse, superordinate, human & 708.50 & 24.36 & 93.6 & .018 \\
\hline
\end{tabular}

(Manuscript received May 22, 2007;

revision accepted for publication November 1, 2007.) 